\title{
The Great Masquerader: Pulmonary Embolism
}

\author{
Syed T Alam* and Syed Tabish Ahsan \\ James Paget University Hospital, UK
}

ISSN: 2639-0531

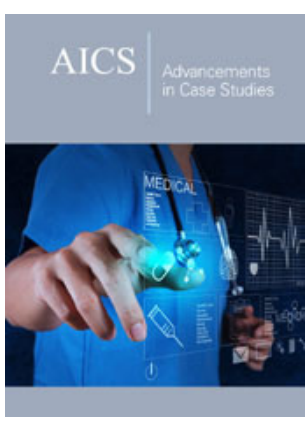

*Corresponding author: Syed T Alam, James Paget University Hospital, UK

Submission: 海 August 29, 2019

Published: 海 September 12, 2019

Volume 2 - Issue 1

How to cite this article: Syed T Alam, Syed Tabish Ahsan. The Great Masquerader: Pulmonary Embolism. Adv Case Stud.2(1) AICS.000529.2019.

DOI:_10.31031/AICS.2019.02.000529

Copyright@ Syed T Alam, This article is distributed under the terms of the Creative Commons Attribution 4.0 International License, which permits unrestricted use and redistribution provided that the original author and source are credited.

\begin{abstract}
We present the case of a 49-year-old male who was referred from Emergency department with worsening breathlessness, chest tightness for last 24 hours. He had a background history of Asthma and Hypertension. Initial ECG revealed symmetric T wave inversions in anterior leads and found to have raised troponin levels. Patient was diagnosed and treated as NSTEMI. While waiting for his coronary angiogram he underwent echocardiogram whose findings were consistent with right sided impairment. Differential diagnosis of Pulmonary Embolism has been made and CT Pulmonary Angiogram done that confirmed diagnosis of Pulmonary Embolism. Early advice from the Respiratory team was sought and patient was treated with rivaroxaban. After a hospital stay, he made a remarkable recovery.
\end{abstract}

Keywords: Acute coronary syndrome; Pulmonary embolism; Rivaroxaban

\section{Introduction}

Acute pulmonary embolism (PE) and Acute Coronary Syndrome (ACS) are considered to be potentially life threatening. Prompt identification of etiology of symptoms can significantly reduce morbidity and mortality. Both the have signs and symptoms that are common to either presentations such as dyspnea, chest discomfort and hemodynamic instability. Electrocardiography (ECG) has been traditionally used to differentiate in the initial stages of work between ACS and PE. More recently T wave inversion has been identified as possible sign of underlying PE. We would like to report a 49-year-old gentleman who presented with chest pain and was initially diagnosed and treated ACS. In this case presentation we would like to present the clinical findings and briefly discuss the salient difference between the two presentations.

\section{Case Description}

This is a 49-year-old gentleman, with well controlled hypertension and asthma, who presented with a two weeks history of generalized fatigue and flu like symptoms along with progressively increasing exertional dyspnea, nausea and chest tightness for the past day.

On Examination, his has a resting heart rate of 98 beats/minute, blood pressure of 118/63, afibrile, respiratory rate of 19 and oxygen saturation of $95 \%$ on room air. His ECG (Figure 1) was consistent with T wave inversions in leads V1-V4 and had elevated troponins at 227 (normal< 40). Based on the work up, patient was initially referred for urgent interventional catheterization for acute coronary syndrome (ACS). Subsequent echocardiogram (video 1) demonstrated a dilated right ventricle (RV) with elevated pressures (Figure 2) with no regional wall motion abnormalities. Given the echocardiographic findings patient underwent a CT angiogram of the chest. He was found to have bilateral pulmonary embolism (PE) (Figure 3). Diagnosis of Pulmonary Embolism was confirmed, and patient was started on direct acting oral anticoagulation (Rivaroxaban). Patient was discharged home and followed by respiratory team. His ensuing echo (after 3 months) showed resolution of RV impairment. As this was unprovoked PE patient was continued on 6 months of anticoagulation

\section{Discussion}

Acute pulmonary embolism (PE) is a highly prevalent condition with a broad clinical presentation, which is principally difficult to diagnose across the emergency departments (EDs) with resulting treatment failure and complications. Mortality in patients with pulmonary 
embolism, who are hemodynamically unstable, is higher than $25 \%$ because of RV dysfunction [1,2]. Acute pulmonary embolism (PE) and Acute Coronary Syndrome (ACS) are common to either presentations such as dyspnea, chest discomfort and hemodynamic instability [3]. Electrocardiography (ECG) has been traditionally used to differentiate in the initial stages of work between ACS and PE.

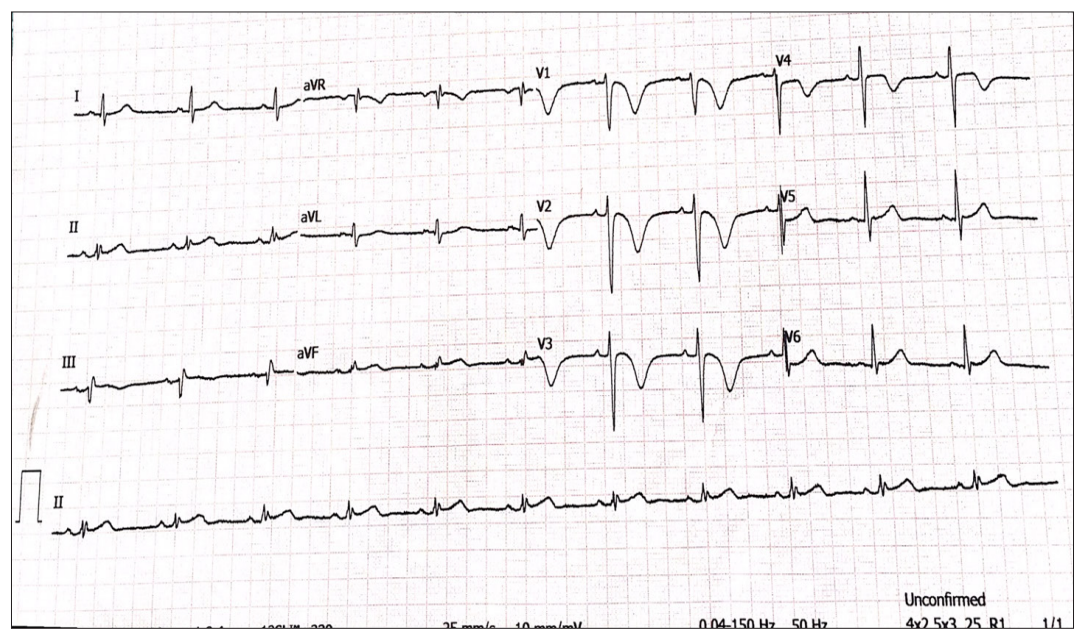

Figure 1: Admitting ECG showing deep symmetric T wave inversions in lead V1-V4.

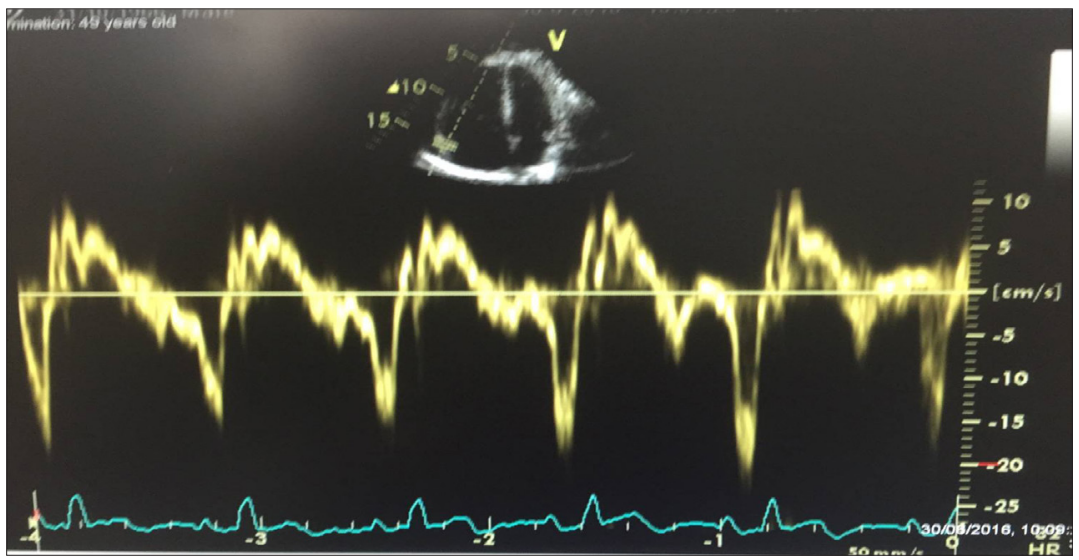

Figure 2: Tissue Doppler study of the tricuspid annulus showing decreased $(7 \mathrm{~cm} / \mathrm{s})$ systolic velocity (s') and moderate right ventricular dysfunction.

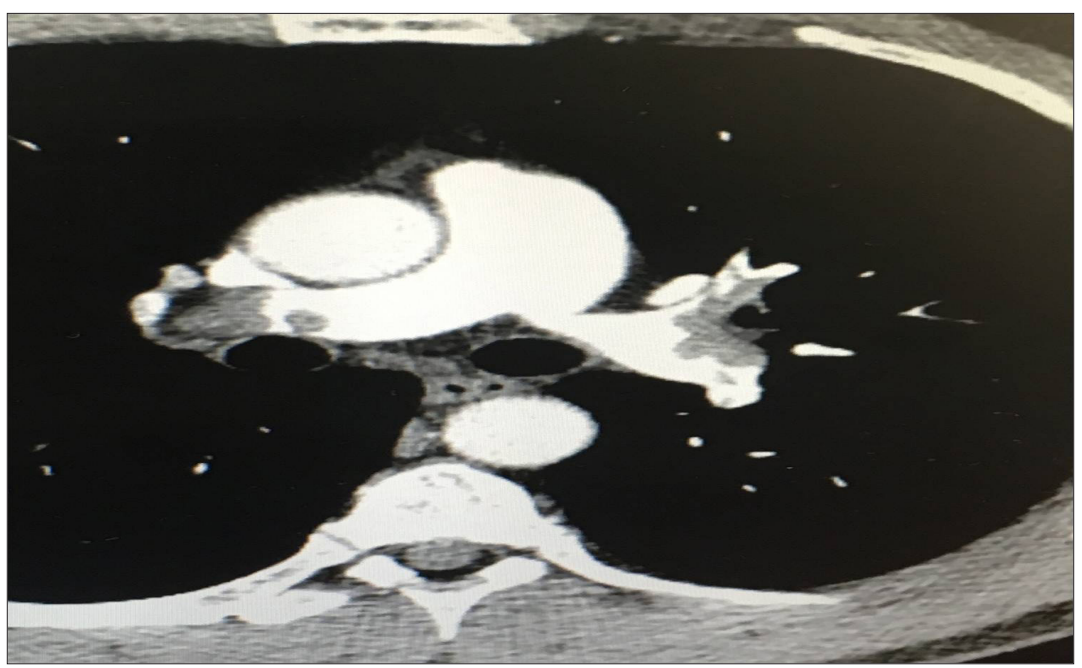

Figure 3: CTPA images consistent with thrombus in both right \& left pulmonary arteries. 
Wellens' syndrome is also characterized by classic T-waves found in precordial leads especially V2-V3. The classic T-waves are described as either deeply inverted or biphasic, especially V2-V3 (may include V1 -V4) during pain-free periods in a patient presenting with ACS [4,5]. Discovering Wellens' syndrome is imperative as $75 \%$ of patients will develop acute anterior wall MI [6,]. These findings are suggestive of a high-grade proximal stenosis of the left anterior descending artery. While both ACS and PE can present with $\mathrm{T}$ wave inversions and elevated troponins, it is critical to differentiate among them in early presentation.

Classical differences between ACS and PE findings on the ECG are as following:

A. PE associated with pulmonary P waves, S1S2S3 pattern, S1Q3T3 pattern, Low voltage and Clockwise rotation (shift in transition zone (R=S) in V5-V6 [7].

B. Negative T waves in lead III and V1 were present only in $1 \%$ of ACS

C. Absence of T wave's inversion in lead 1 \& a VL and presence in V1 is highly suggestive of Pulmonary Embolism.

\section{Conclusion}

This case study implicates the importance of holistic approach to the patients. Furthermore, early echocardiogram in patients presenting with chest pain might lead to improved and good outcomes. Lastly, this case specifically reminds a healthcare professional that even though acute coronary syndrome may be evident with ECG, some number of the patients do present with a more concomitant or other disease that increases the complication.

\section{References}

1. Goldhaber SZ, Visani L, De Rosa M (1999) Acute pulmonary embolism: clinical outcomes in the International Cooperative Pulmonary Embolism Registry (ICOPER). Lancet 353(9162):1386-1389.

2. Kasper W, Konstantinides S, Geibel A, Olschewski M, Heinrich F, et al. (1997) Management strategies and determinants of outcome in acute major pulmonary embolism: results of a multicenter registry. J Am Coll Cardiol 30(5): 1165-1171.

3. Fedullo PF, Tapson VF (2003) The evaluation of suspected pulmonary embolism. N Eng J Med 349(13): 1247-12561.

4. De Zwaan C, Bar FW, Wellens HJ (1982) Characteristic electrocardiographic pattern indicating a critical stenosis high in left anterior descending coronary artery in patients admitted because of impending myocardial infarction. Am Heart J 103: 730-736.

5. Movahed MR (2008) Wellen's Syndrome or Inverted U-waves? Clin Cardiol 31(3): 133-134.

6. Smith S, Whitwam S (2006) Acute coronary syndromes. Emerg Med Clin N Am 24(1): 53-89.

7. Sukhija R, Aronow WS, Ahn C, Kakar P (2006) Electrocardiographic abnormalities in patients with right ventricular dilation due to acute pulmonary embolism. Cardiology 105(1): 57-60. 